\title{
Weekly Cyclodextrin Administration Normalizes Cholesterol Metabolism in Nearly Every Organ of the Niemann-Pick Type C1 Mouse and Markedly Prolongs Life
}

\author{
CHARINA M. RAMIREZ, BENNY LIU, ANNA M. TAYLOR, JOYCE J. REPA, DENNIS K. BURNS, ARTHUR G. WEINBERG,
} STEPHEN D. TURLEY, AND JOHN M. DIETSCHY

\begin{abstract}
Departments of Pediatrics [C.M.R.], Internal Medicine [B.L., S.D.T., J.J.R., J.M.D.], Physiology [A.M.T., J.J.R.], and Pathology [D.K.B.,
\end{abstract} A.G.W.], University of Texas Southwestern Medical School at Dallas, Dallas, Texas 75390

\begin{abstract}
Niemann-Pick type C1 (NPC1) disease arises from a mutation inactivating NPC1 protein that normally moves unesterified cholesterol from the late endosomal/lysosomal complex of cells to the cytosolic compartment for processing. As a result, cholesterol accumulates in every tissue of the body causing liver, lung, and CNS disease. Treatment of the murine model of this disease, the $n p c 1^{-1-}$ mouse, s.c. with $\beta$-cyclodextrin $(4000 \mathrm{mg} / \mathrm{kg})$ one time each week normalized cellular cholesterol metabolism in the liver and most other organs. At the same time, the hepatic dysfunction seen in the untreated $n p \mathrm{cl}^{-1-}$ mouse was prevented. The severity of cerebellar neurodegeneration also was ameliorated, although not entirely prevented, and the median lifespan of the animals was doubled. However, in contrast to these other organs, lung showed progressive macrophage infiltration with development of lipoid pneumonitis. These studies demonstrated that weekly cyclodextrin administration overcomes the lysosomal transport defect associated with the NPC1 mutation, nearly normalizes hepatic and whole animal cholesterol pools, and prevents the development of liver disease. Furthermore, this treatment slows cerebellar neurodegeneration but has little or no effect on the development of progressive pulmonary disease. (Pediatr Res 68: 309-315, 2010)
\end{abstract}

$\mathrm{N}$ iemann-Pick type $\mathrm{C} 1$ (NPC1) disease arises when a mutation inactivates the protein NPC1, which normally operates in association with NPC2 to move unesterified cholesterol $(\mathrm{C})$ across the limiting membrane of the late endosomal/lysosomal (E/L) complex of cells to the cytosolic compartment where it can be metabolized and/or excreted (1). In the absence of NPC1 function, $\mathrm{C}$ continuously accumulates in every tissue of the body at rates proportional to the uptake of $\mathrm{apoB}_{100}$ and apoE containing lipoproteins by each organ through receptor-mediated and bulk-phase endocytosis $(2,3)$. In the murine model of this disease, the NPC1 mouse, this leads to expansion of the whole animal cholesterol pool by $70-80 \mathrm{mg} / \mathrm{d}$ per kg body weight. In both mouse and child, this accumulation of $\mathrm{C}$ is associated with macrophage infiltration

Received March 24, 2010; accepted June 16, 2010.

Correspondence: John M. Dietschy, M.D., Department of Internal Medicine, University of Texas Southwestern Medical Center at Dallas, Dallas, TX 75390-9151; e-mail: john.dietschy@utsouthwestern.edu

Supported by US Public Health Service Research Grant R01-HL009610 [J.M.D, S.D.T.] and by grants from the Moss Heart Fund [J.M.D.] and the Ara Parseghian Medical Research Foundation [J.J.R.]. C.M.R. and B.L. also received postdoctoral support from the Ara Parseghian Medical Research Foundation.

C.M.R. and B.L. contributed equally to this work. and activation and with parenchymal cell death in many organs including the liver, lung and CNS (3-5). Clinically, these histological alterations are manifested as enlargement of the liver and spleen, active liver disease, lipoid pneumonitis, and progressive neurological dysfunction.

One approach to the treatment of this genetic disorder is to limit the delivery of sterol to the target tissues which, in turn, would reduce the amount of $\mathrm{C}$ that is sequestered in a particular organ and ameliorate cell death and severity of disease. For example, blocking the intestinal absorption of sterol in the NPC1 mouse with a drug like ezetimibe reduces the flow of cholesterol to the liver through uptake of chylomicron remnants. As a consequence, the size of the liver and the amount of $\mathrm{C}$ sequestered are reduced, and there is an improvement in liver function abnormalities and molecular measures of inflammation (6). Alternatively, treatment with an agent such as an LXR agonist increases cholesterol loss from the CNS, presumably reducing the amount of apoE/C complex available for uptake into glia and neurons $(7,8)$. This, in turn, is associated with a reduction in glial activation and measures of inflammation and with amelioration of the neurodegeneration (7).

A second, more effective approach to this disorder comes from the observation that administration of the cholesterolbinding agent, 2-hydroxypropyl- $\beta$-cyclodextrin (CYCLO), to the mutant mouse in vivo allows the $\mathrm{C}$ sequestered in the late $\mathrm{E} / \mathrm{L}$ compartment of cells throughout the body to immediately move to the cytosolic compartment and be metabolized through normal pathways for excretion from the animal (9). After a single s.c. dose of CYCLO, in nearly every organ, there is a rapid increase in the concentration of cholesteryl esters (CE), suppression of SREBP2 target genes and C synthesis, and activation of several LXR target genes. These changes all attest to the fact that the sequestered pool of $\mathrm{C}$ has rapidly moved to the metabolically active pool of sterol in the cytosolic compartment of cells. Over the next several days, this pool of excess cholesterol that is temporarily stored in the cytosolic compartment as CE moves to the liver and is excreted from the body, predominantly as fecal acidic sterols

\footnotetext{
Abbreviations: C, unesterified cholesterol; CE, cholesteryl esters; CYCLO, 2-hydroxypropyl- $\beta$-cyclodextrin; E/L, endosomal/lysosomal; NPC1, NiemannPick type $\mathrm{C} 1$
} 
(10). When CYCLO is administered to the mutant animal, either as a single dose or as repeated doses, the liver disease and neurodegeneration are significantly improved, and life expectancy is extended $(6,9,11)$. This striking effect of CYCLO in acutely reversing or bypassing the transport defect present in the limiting membrane of the late $\mathrm{E} / \mathrm{L}$ complex provides an explanation for how the sequestered pool of $\mathrm{C}$ can be metabolized and excreted from the animal.

This observation, in turn, raises the important possibility that repeated doses of this compound might totally normalize whole animal cholesterol pools and prevent clinical disease in the NPC1 mouse. This is particularly likely given the recent observations that the young NPC1 mouse over the course of a week sequesters $\sim 490-560 \mathrm{mg} / \mathrm{kg}$ of excess $\mathrm{C}$ while a single dose of CYCLO can flush from the body $\sim 500 \mathrm{mg} / \mathrm{kg}$ (9). Thus, the present studies were undertaken, first, to determine whether weekly CYCLO administration could entirely prevent expansion of the whole animal sterol pool and, further, whether this therapeutic effect would be seen equally in every organ. Second, additional investigations were undertaken to determine whether this reduction in tissue cholesterol content correlated with histological and clinical evidence of amelioration of disease and with prolongation of life.

\section{MATERIALS AND METHODS}

\begin{abstract}
Animals. Control $\left(n p c 1^{+/+}\right)$and homozygous mutant $\left(n p c 1^{-/-}\right)$mice were generated from heterozygous $\left(n p c 1^{+/-}\right)$animals on a pure BALB/c background, and the pups were genotyped at $19 \mathrm{~d}$ of age $(1,2)$. All experimental protocols were approved by the Institutional Animal Care and Use Committee of The University of Texas Southwestern Medical School.

Diets and treatment. Animals were fed ad libitum a cereal-based, lowcholesterol (0.02\% cholesterol, $4 \%$ total fat, wt/wt) diet (no. 7001; Harland Teklad, Madison, WI) on weaning. Groups of mice were administered a s.c. injection of a $20 \%$ (wt/vol, in saline) solution of CYCLO (product H107; Sigma Chemical Co.), $4000 \mathrm{mg} / \mathrm{kg}$ body weight, during the late dark phase $(0900 \mathrm{~h})$ at the scruff of the neck (12). Matching mice were also injected with saline alone to serve as controls.

Tissue cholesterol concentrations and synthesis rates. Tissue cholesterol concentrations and synthesis rates were determined as described (10), as were liver triacylglycerol contents (13). Esterified cholesterol levels in tissues were also measured as described (6). Tissue sterol contents are expressed as milligrams of cholesterol per whole organ (mg/organ). The cholesterol values found in every tissue, and in the remaining carcass, were summed to give whole animal cholesterol pools expressed as milligrams of cholesterol per $\mathrm{kg}$ body weight $(\mathrm{mg} / \mathrm{kg})$. The rates of sterol synthesis are expressed as the amount of $\left[{ }^{3} \mathrm{H}\right]$ water incorporated into sterols per hour per organ $(\mathrm{nmol} / \mathrm{h} /$ organ). The $\left[{ }^{3} \mathrm{H}\right]$ water incorporation rates in all organs were summed and used to calculate the whole animal synthesis rates. These values were then converted to the absolute $\mathrm{mg}$ of cholesterol synthesized each day per kg body weight $(\mathrm{mg} / \mathrm{d} / \mathrm{kg})$.
\end{abstract}

Relative mRNA levels. Tissues from each animal were collected, snapfrozen in liquid nitrogen, and stored at $-85^{\circ} \mathrm{C}$. Quantitative real-time PCR was performed using an Applied Biosystems 7900HT sequence detection system and SYBR-green chemistry (14).

Liver function tests. Plasma was sent to a commercial laboratory for measurement of alanine aminotransferase (ALT), aspartate aminotransferase (AST), and alkaline phosphatase.

Tissue histology. Tissues sections were prepared, and histological examination and Purkinje cell number were assessed as described (9).

Data analysis. All data are presented as a mean \pm 1 SEM. Differences between means in groups were tested for significance $(p<0.05)$ using one-way ANOVA, followed by the Newman-Keuls multiple comparison test (Graph-Pad Software, Inc., San Diego, CA). Significant differences between groups are designated with different symbols. Statistical differences among survival curves were determined using the Wilcoxon-Gehard and Log-rank analyses.

\section{RESULTS}

Groups of $n p c 1^{+/+}$and $n p c 1^{-/-}$mice were given either saline or a dose of CYCLO $(4000 \mathrm{mg} / \mathrm{kg})$ s.c. at $7 \mathrm{~d}$ of age and every week thereafter until they were studied as young adults at $49 \mathrm{~d}$ of age. At this time, the $n p c 1^{+/+}$and $n p c 1^{-/-}$mice treated with only saline weighed $21.8 \pm 1.0$ and $20.0 \pm 0.8 \mathrm{~g}$, respectively. As seen in Figure $1 A-F$, the $n p c 1^{-/-}$mice receiving only saline had significant enlargement of the liver, spleen, and lung but not of the other organs. Weekly treatment with CYCLO prevented this hepatosplenomegaly.

Cholesterol content and synthesis. Except for the brain, the $\mathrm{C}$ content was markedly elevated in all organs of the saline treated $n p c 1^{-/-}$mice (Fig. $1 G-L$ ). Although $\mathrm{C}$ does accumulate in glia and neurons in the brain of the $\mathrm{npcl}^{-/-}$mice, this accumulation is offset by loss of $\mathrm{C}$ through partial demyelination $(15,16)$. Importantly, nearly all of this sequestered sterol was $\mathrm{C}$ because the content of $\mathrm{CE}$ in every organ was very low (Fig. $1 M-R$ ). The rate of sterol synthesis was also significantly increased in every organ, except the brain, as previously described (Fig. $1 S-X)(9,15)$.

However, after weekly CYCLO administration to the $n p c 1^{-1-}$ mice, these abnormalities in tissue cholesterol metabolism were all largely prevented. The content of $\mathrm{C}$ in nearly all organs of these treated animals was markedly reduced. There was no increase in the content of CE in any organ, and the rates of sterol synthesis were maintained at or near the levels found in the animals with normal NPC1 function, even in the brain. The notable exception to these findings was the lung where there was still a significant increase in $\mathrm{C}$ content (Fig. $1 J$ ) and synthesis (Fig. 1V).

Relative mRNA levels for macrophage markers. Histologically, one of the hallmarks of NPC1 dysfunction and disease is a striking macrophage infiltration (3). This abnormality was reflected in the markedly elevated expression of mRNA for the macrophage and monocyte markers, CD68 and CD11c, and the cytokine, TNF $\alpha$, found in the brain, liver, and lung of the untreated $n p c 1^{-/-}$mice (Fig. 2). However, with weekly CYCLO administration, these abnormalities were near completely prevented in the brain and liver. However, the lung was resistant to this treatment (Fig. $2 C$ and $F$ ).

Whole animal pools and clinical effects. The whole mouse cholesterol pool in the $n p c l^{+/+}$animals was $\sim 2400 \mathrm{mg} / \mathrm{kg}$, regardless of whether these mice were administered saline or CYCLO, whereas this pool reached $5408 \mathrm{mg} / \mathrm{kg}$ in the untreated $n p c 1^{-/-}$animals (Fig. 3A). Weekly CYCLO administration kept this pool at almost normal levels $(2811 \mathrm{mg} / \mathrm{kg})$. Similarly, the rate of whole animal cholesterol synthesis was nearly doubled $(238 \mathrm{mg} / \mathrm{d} / \mathrm{kg})$ in the mutant mice, but this rate was maintained at near normal levels $(137 \mathrm{mg} / \mathrm{d} / \mathrm{kg})$ with weekly CYCLO administration (Fig. $3 B$ ). The $n p c 1^{-1-}$ mice had markedly abnormal liver function tests (Fig. $3 C-E$ ), depressed levels of hepatic triacylglycerol (Fig. $3 F$ ), and elevated plasma cholesterol concentrations (Fig. $3 G$ ). All of these functional and lipid abnormalities were prevented by weekly CYCLO administration to the $n p c 1^{-/-}$mice. Finally, in the 49-d-old mutant animals, $\sim 75 \%$ of the Purkinje cells in the 


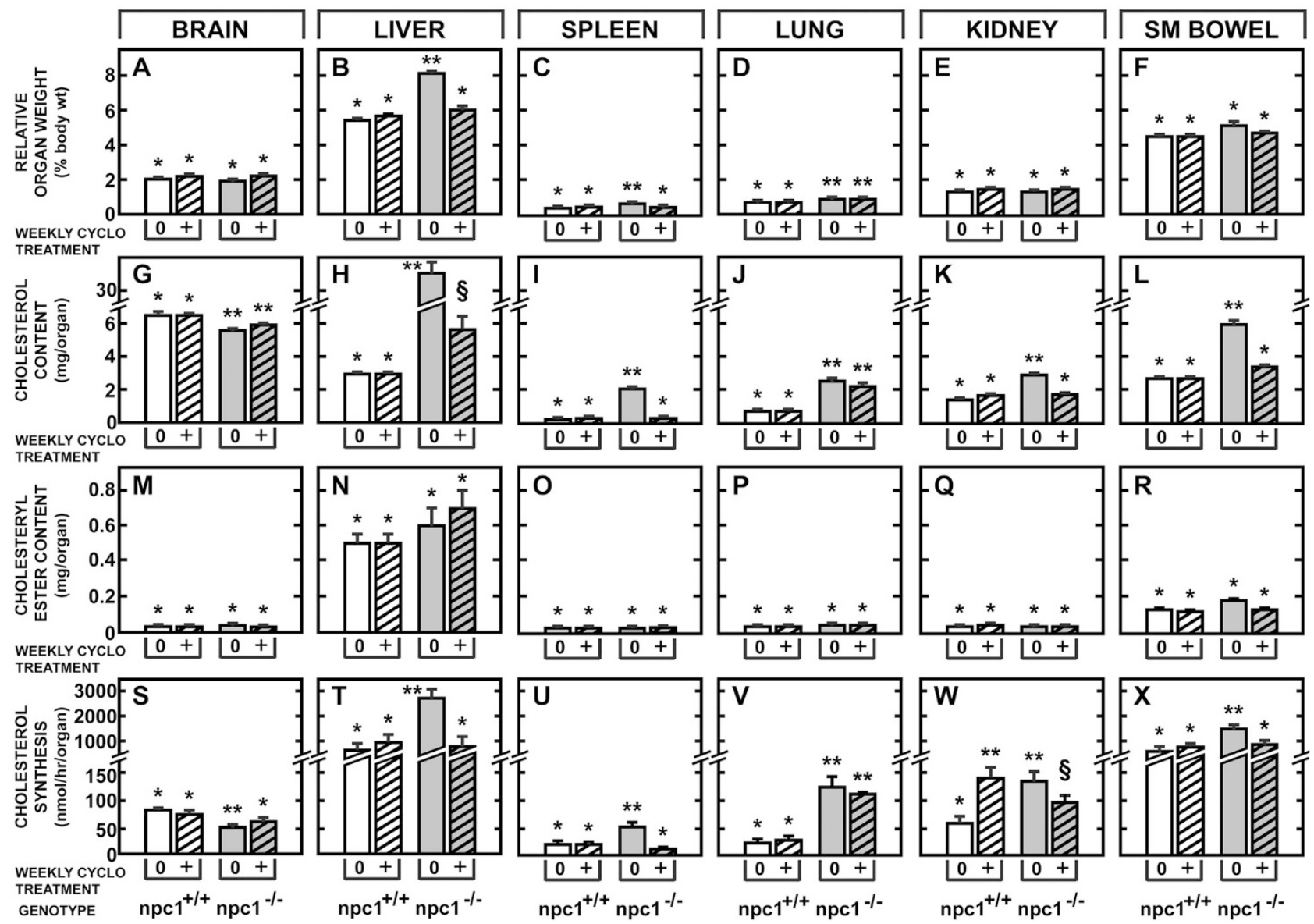

Figure 1. Effect of weekly CYCLO administration on organ weights and various parameters of cholesterol metabolism in the $n p c 1^{--}$mouse. Both $n p c 1^{+/+}$ and $n p c 1^{-/-}$mice were treated s.c., weekly with either saline or CYCLO $(4,000 \mathrm{mg} / \mathrm{kg})$, and then studied at $49 \mathrm{~d}$ of age. The relative organ weights were measured in six major tissues $(A-F)$. The content of total cholesterol present in each of these tissues was quantitated $(G-L)$ as was the component of this sterol pool that was esterified $(M-R)$. Both of these latter values are given as the milligrams of sterol present in the whole organs. Cholesterol synthesis rates in these same tissues are also shown $(S-X)$ and are expressed as the nmol of $\left[{ }^{3} \mathrm{H}\right]$ water incorporated into sterols per hour per organ. Each column represents the mean \pm 1 SEM for six animals. Significant differences $(p<0.05)$ among groups are designated by different symbols in each panel.

cerebellum had disappeared, (Fig. 3H), but after CYCLO administration, nearly twice as many of these cells survived.

Animal survival. To explore the overall effect of these changes on longevity, groups of $n p c 1^{-/-}$mice were administered either saline alone, CYCLO one time at $7 \mathrm{~d}$ of age, or CYCLO at $7 \mathrm{~d}$ of age followed by serial, weekly injections. As seen in Figure 4, the single dose of CYCLO at $7 \mathrm{~d}$ of age significantly prolonged life by about $30 \mathrm{~d}$, while continuous weekly administration of this compound extended lifespan by $\sim 80 \mathrm{~d}$. Of note, this latter group of mice seemed very mobile and vigorous, even beyond $120 \mathrm{~d}$ of age, and the $n p \mathrm{Cl}^{-/-}$ females could be readily mated with $n \mathrm{pcl}^{+/+}$males, the $n p c 1^{-/-}$males with $n p c 1^{+/+}$females, giving rise to heterozygous offspring.

Histology of the major target organs. The histology of several important organs in the various experimental groups is shown in Figure 5. The first three columns show organs taken from 49-d-old $n p c 1^{+/+}$mice given only saline and from $n p c 1^{-/}$animals administered either saline or CYCLO every week. The last two columns of figures come from 160-d-old $n p c 1^{-/-}$and $n p c 1^{+/+}$mice treated weekly with CYCLO. At $49 \mathrm{~d}$ of age, only $\sim 25 \%$ of Purkinje cells remained in the untreated $n p \mathrm{Cl}^{-1-}$ mice, and many of these seemed to be pyknotic and dying (Fig. 5B). CYCLO treatment slowed this rate of Purkinje cell loss, but still cell numbers were significantly reduced (Fig. 5C). Importantly, in animals that survived to $160 \mathrm{~d}$ of age, no Purkinje cells could be identified (Fig. 5D). In untreated 49 -d-old $n p c 1^{-\prime-}$ mice, there were numerous lipid-laden macrophages scattered throughout the liver (Fig. $5 G$ ), whereas this infiltrate was almost completely absent in the animals treated with CYCLO (Fig. 5H). Even after $160 \mathrm{~d}$ of treatment, the architecture of the liver was essentially normal in the $n p c 1^{-1-}$ mice, except for occasional clusters of macrophages in a pericentral distribution (Fig. 5I). The histological appearance of the liver was entirely normal in $n p c 1^{+/+}$ animals treated with weekly CYCLO (Fig. $5 J$ ).

The lung behaved differently, however. In the 49-d-old, untreated $\mathrm{npcl}^{-/-}$mice, there were small clusters of macrophages scattered throughout the alveoli of the lung (Fig. 5L), and similar clusters were still found after treatment with CYCLO (Fig. 5M). This progressive infiltration continued, even with weekly CYCLO treatment, until at $160 \mathrm{~d}$ of age there were collections of lipid-laden macrophages filling many of the alveolar spaces (Fig. $5 N$ ). Of note, the lungs seemed to be entirely normal in the $\mathrm{npCl}^{+/+}$mice treated with weekly CYCLO (Fig. 5O). Finally, kidney architecture was essen- 


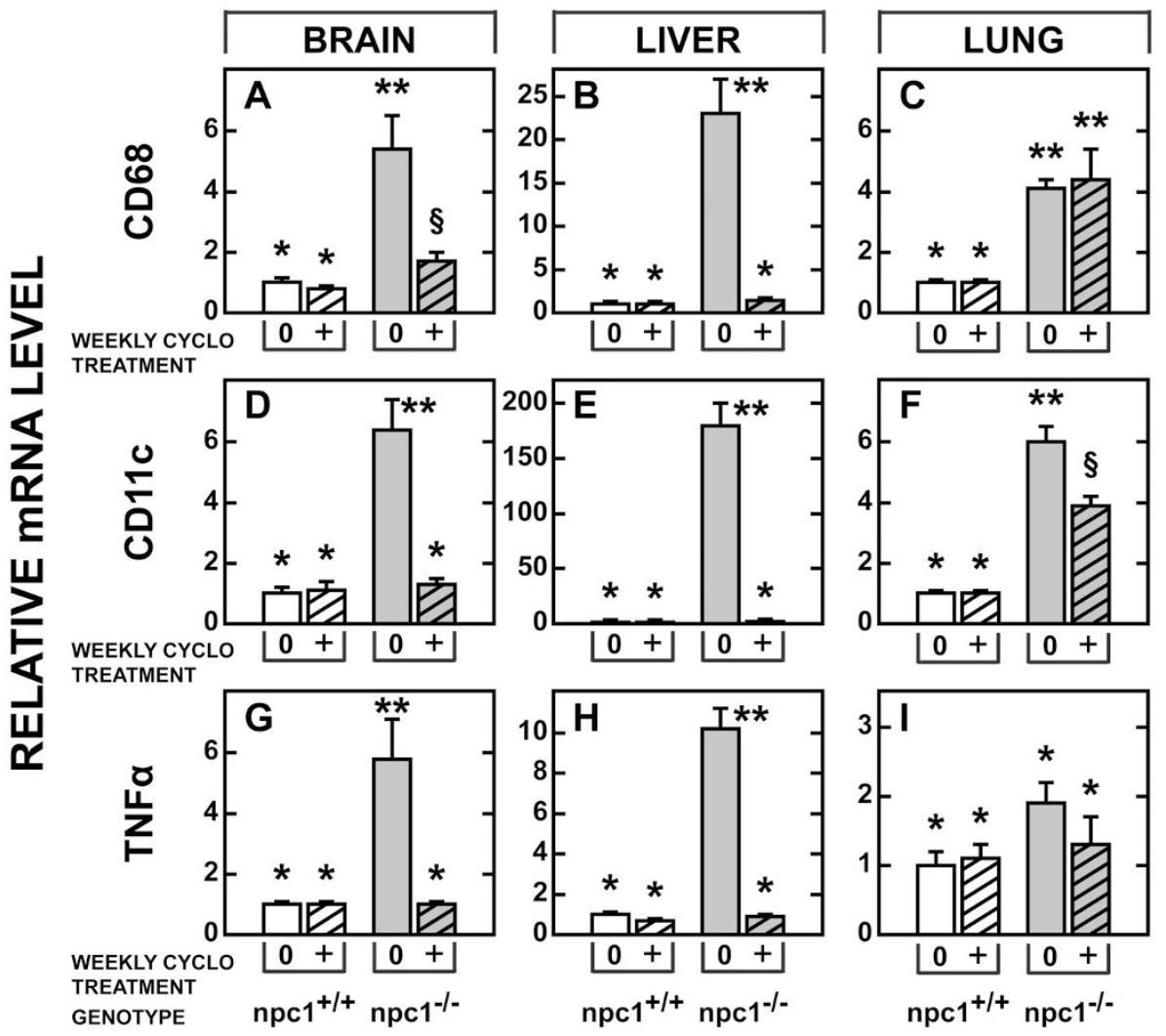

Figure 2. Effect of weekly CYCLO administration on the relative mRNA levels of various inflammatory proteins in brain, liver, and lung. Both $n p c 1^{+/+}$and $n p c 1^{-/-}$mice were treated weekly with either saline or CYCLO and then studied at $49 \mathrm{~d}$ of age. Each column represents the mean \pm 1 SEM for six mice. Significant differences $(p<0.05)$ among groups are indicated by different symbols in each panel. tially normal in the untreated $n p c 1^{-/-}$mice (Fig. $5 Q$ ), but vacuolization was noted in some tubular epithelium after the administration of CYCLO (Fig. 5R), as has been previously reported (17). Of note, this vacuolization apparently did not progress even after $160 \mathrm{~d}$ of weekly CYCLO treatment (Fig. $5 S$ and $T)$.

Flow of $C$ from the late E/L complex to the cytosol. Finally, there is a remarkable difference in CE formation and suppression of cholesterol synthesis in these studies using repetitive CYCLO administration when compared with previously published investigations using a single dose of this cholesterol-binding agent. These differences go directly to the mechanism of action of CYCLO within the cells of the body. Although 49-d-old $n p c 1^{-1-}$ mice not previously treated with CYCLO had markedly expanded pools of sequestered $\mathrm{C}$ in nearly every organ (Fig. $3 A$ ), these pools were largely maintained at normal levels after weekly administration of this compound. As a result of these differences, acute reversal of the transport defect in the untreated $\mathrm{npcl}^{-/-}$mice led to flux of massive amounts of $\mathrm{C}$ into the cytosolic compartment, whereas there was virtually no increase in this flux in animals previously treated with weekly CYCLO administration. These differences are illustrated by the data shown in Figure 6. In 48-d-old, $n p \mathrm{pl}^{-/-}$mice that had received no early CYCLO treatment, the pools of tissue $\mathrm{C}$ were markedly elevated in liver (9-fold), spleen (7-fold), and kidney (2-fold) and rates of synthesis were increased. Twenty-four hours after administration of a single dose of CYCLO, CE levels had markedly increased in the liver (15-fold), spleen (9-fold), and kidney (2-fold; Fig. 6A, $C$, and $E$ ), whereas synthesis was markedly suppressed (Fig. $6 G, I$, and $K$ ). Thus, these indirect measures of $\mathrm{C}$ flow into the cytosolic compartment demonstrated a metabolic effect proportional to the size of the pool of $\mathrm{C}$ sequestered in each organ. In contrast, if this sequestration was prevented by earlier weekly administration of CYCLO and the tissue $\mathrm{C}$ pools were relatively normal in the liver (1.9-fold), spleen (1.1-fold), and kidney (1.3-fold), then 24 hours after CYCLO administration, there was little or no increase in the level of $\mathrm{CE}$ in these organs (Fig. $6 B, D$, and $F$ ) nor was there significant suppression of synthesis (Fig. $6 H, J$, and $L$ ). Thus, repetitive CYCLO administration had markedly reduced the pool of $\mathrm{C}$ in the late $\mathrm{E} / \mathrm{L}$ compartment of cells and so markedly blunted the metabolic response of these cells to acute CYCLO administration. CYCLO had no effect on tissues of the $n p c 1^{+/+}$or $n p c 1^{-/-}$mice treated weekly with this compound because there were no pools of sequestered, excess $\mathrm{C}$ in the tissues of these animals.

\section{DISCUSSION}

These studies demonstrate that weekly CYCLO administration almost prevents the abnormal cholesterol sequestration and tissue metabolic dysfunction typical of NPC1 disease (Figs. 1 and 3). As a result, plasma cholesterol concentrations, hepatic triacylglycerol levels, liver function tests, and measures of tissue macrophage infiltration and inflammation are all maintained at normal levels. In addition, the magnitude of neurodegeneration is slowed, animal fertility is restored, and lifespan of the $n p \mathrm{nl}^{-/-}$animals is essentially doubled. These findings support the concept that it is the disordered $\mathrm{C}$ metab- 


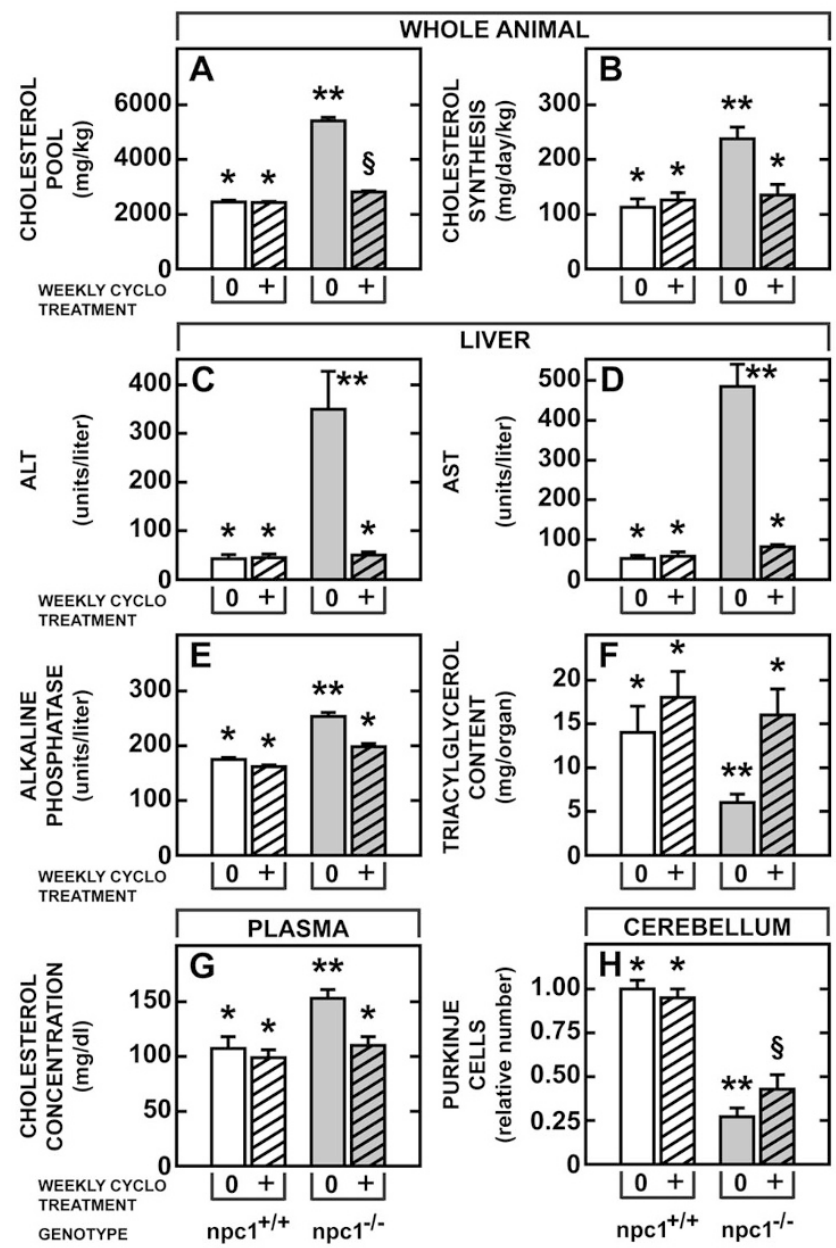

Figure 3. Effect of weekly CYCLO administration on whole animal cholesterol metabolism, liver function tests, plasma total cholesterol concentrations, and cerebellar neurodegeneration. Both $n p c 1^{+/+}$and $n p c 1^{-/-}$mice were treated weekly with either saline or CYCLO and then studied at $49 \mathrm{~d}$ of age. Whole animal cholesterol pools are expressed as milligrams of cholesterol per $\mathrm{kg}$ body weight $(A)$. Whole animal synthesis rates $(B)$ are expressed as milligrams of cholesterol synthesized per day per kg body weight. The liver function tests $\operatorname{ALT}(C)$, AST $(D)$, and alkaline phosphatase $(E)$ were quantitated, as were the levels of hepatic triacylglycerol $(F)$. The plasma total cholesterol concentrations were also measured $(G)$. The numbers of Purkinje cells in the cerebellum were counted and are expressed relative to the number found in the $n p \mathrm{Cl}^{+/+}$mice treated with saline $(H)$. Each column represents the mean \pm 1 SEM for six animals in each group. Significant differences $(p<$ 0.05 ) among groups are indicated by different symbols in each panel.

olism in organs of the NPC1 mouse, and child, that leads, directly or indirectly, to organ dysfunction and disease.

At the dose used in these studies, plasma concentrations of CYCLO reach $2-5 \mathrm{mg} / \mathrm{mL}$ within $1-3 \mathrm{~h}$ after administration. The compound is then rapidly excreted in the urine, and virtually no $\mathrm{C}$ is carried out of the animals with the excreted CYCLO (10). Rather, in cells throughout the body, within hours of administration, there is net flow of the sequestered pool of $\mathrm{C}$ out of the late $\mathrm{E} / \mathrm{L}$ compartment into the cytosol where it is temporarily stored as $\mathrm{CE}(9,10)$. Over the following few days, this pool of CE in the peripheral organs is slowly hydrolyzed, and the released $\mathrm{C}$ is moved across the plasma membrane onto HDL and transferred to the liver. This process of centripetal sterol flow is unaffected by the mutation in

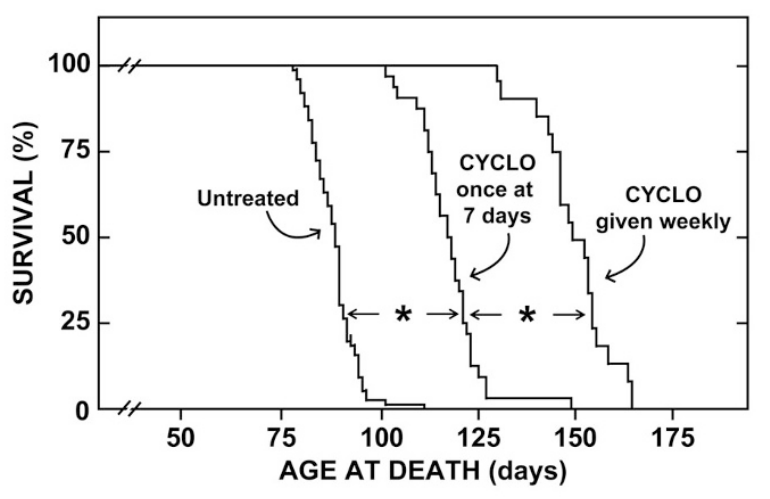

Figure 4. Age at death of $n p c 1^{-/-}$mice treated with CYCLO. This figure shows three groups of $n p c 1^{-1-}$ animals that were either untreated, administered a single dose of CYCLO $(4000 \mathrm{mg} / \mathrm{kg})$ once at $7 \mathrm{~d}$ of age, or treated weekly with this same dose of CYCLO. There were 76 untreated mice, 32 treated once, and 20 treated weekly. Both the single and weekly doses of CYCLO significantly (*) prolonged the life of these $n p c 1^{-/-}$animals $(p<$ 0.001).

NPC1 (18). The pool of C generated from the hydrolysis of $\mathrm{CE}$ in the liver, along with that taken up from HDL, is then metabolized to bile acid and excreted from the body as fecal acidic sterols (10). In this manner, the weekly administration of CYCLO permits excretion of an amount of C essentially equal to that ordinarily sequestered in the individual organs of the $n \mathrm{pll}^{-/-}$mouse and, so, maintains the whole animal cholesterol pool at near normal levels (Fig. 3A).

However, it is clear from these studies that not all organs respond equally well to the s.c. administration of CYCLO. In the liver, for example, a single dose of CYCLO given to young or mature $n \mathrm{pcl}^{-/-}$mice leads to the acute flow of $\mathrm{C}$ into the cytosolic compartment for metabolism and/or excretion (10). As a result of this responsiveness to a single dose, the weekly administration of CYCLO maintains essentially normal hepatic $\mathrm{C}$ pools and synthesis rates (Fig. 1), prevents infiltration of lipid-laden macrophages (Fig. 5), maintains molecular markers of inflammation at normal levels (Fig. 2), and prevents abnormalities in liver function tests and liver disease (Fig. 3). Thus, in the liver, and many other organs, weekly CYCLO administration nearly completely overcomes the transport defect seen in the $n p c l^{-1-}$ animals.

However, the response in the brain is less robust, and s.c. administration of a single dose of CYCLO to either 7-d-old or mature $n \mathrm{pCl}^{-/-}$animals also results in the flow of $\mathrm{C}$ into the cytosolic CE pool and suppression of sterol synthesis (10). However, the magnitude of this response is quantitatively less than that seen in liver. Thus, with the weekly administration of CYCLO used in these studies, there is only partial restoration of $\mathrm{C}$ metabolism in the brain, although measures of glial cell activation are maintained at near normal levels (Fig. 2). Associated with these changes, the rate of Purkinje cell death is slowed (Figs. 3 and 5), and the animals survive for a much longer time without gross neurological abnormalities (Fig. 4). This partial response of the brain to systemic CYCLO administration is the result of lower permeability of the capillaries of the brain to this compound. 


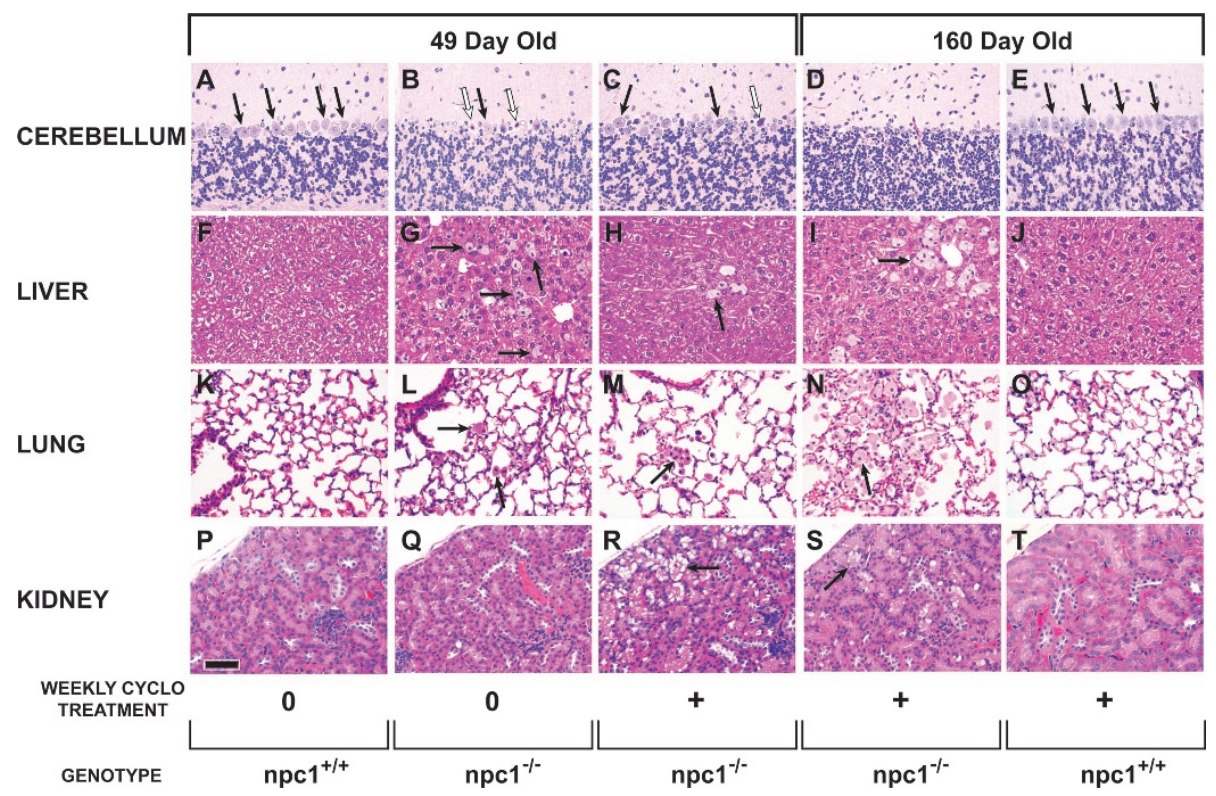

Figure 5. Representative histological sections of four tissues in $n p c 1^{-/-}$mice treated with CYCLO. Both $n p c 1^{+/+}$and $n p c 1^{-/-}$mice were administered saline, whereas other groups of animals were given CYCLO $(4,000 \mathrm{mg} / \mathrm{kg})$ weekly. Histological preparations were then made of the cerebellum, liver, lung, and kidney at either 49 or $160 \mathrm{~d}$ of age. The solid arrows point to Purkinje cells in the cerebellum $(A-E)$, to lipid-laden macrophages in the liver and lung $(F-O)$, or to vacuolization in the kidney epithelium $(P-T)$. The open arrows in panels $\mathrm{B}$ and $\mathrm{C}$ point to pyknotic Purkinje cells. The bar equals $50 \mu \mathrm{m}(P)$, and all panels are at the same magnification $(\times 400)$.
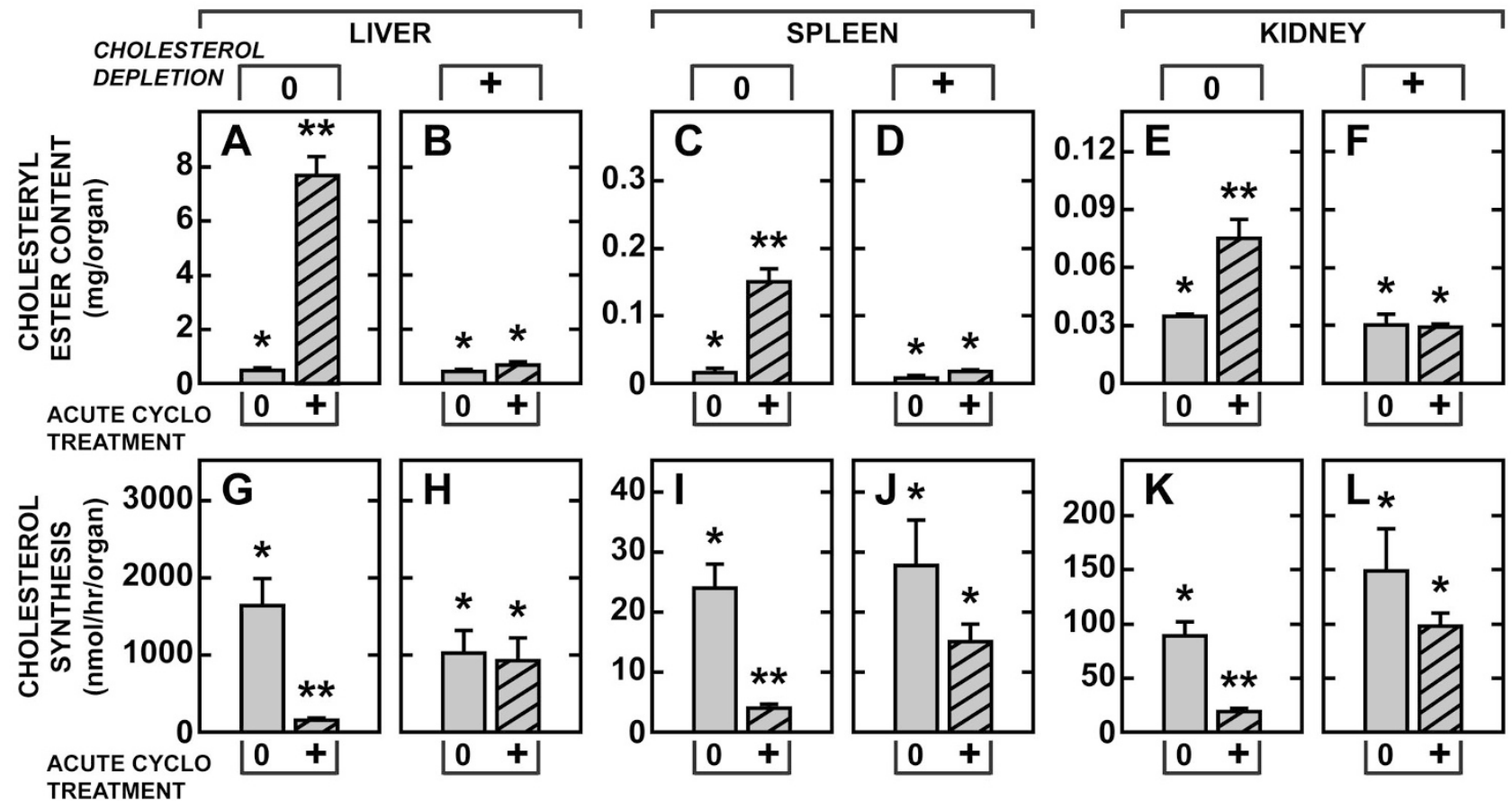

Figure 6. Flow of cholesterol from the late E/L compartment into the cytosolic compartment in $n p c 1^{-/-}$mice that were either untreated or were depleted of sequestered sterol pools. Two groups of $n p c 1^{-1-}$ mice were used in these studies. One group received no early treatment until $48 \mathrm{~d}$ of age, the time at which they were given a single dose of either saline $(0)$ or CYCLO $(4000 \mathrm{mg} / \mathrm{kg})(+)$ and then studied $24 \mathrm{~h}$ later $(A, C, E, G, I, K)$. The second group received weekly CYCLO administration beginning at $7 \mathrm{~d}$ of age to deplete tissue cholesterol pools. At $48 \mathrm{~d}$ of age, these animals were then given either saline (0) or CYCLO $\left(^{+}\right)$and studied $24 \mathrm{~h}$ later $(B, D, F, H, J, L)$. The level of cholesteryl ester found in the liver, spleen, and kidney was quantitated $(A-F)$ as was the rate of cholesterol synthesis in these organs $(G-L)$. Each column represents the mean \pm 1 SEM for four to eight animals in each group. Significant differences $(p<0.05)$ among groups are indicated by different symbols in each panel.

The lung is even more resistant to CYCLO administration than brain. As in the other tissues, the severity of pulmonary disease is proportional to the amount of $\mathrm{C}$ sequestered in the tissue. For example, when uptake of lipoprotein-C is enhanced and the amount of $\mathrm{C}$ sequestered in the lung is increased, there is significantly greater infiltration by lipid-laden macrophages and pneumonitis (3). However, the acute administration of a single dose of CYCLO to $n p c 1^{-/-}$mice has no effect on these pools of $\mathrm{C}$ or on sterol synthesis at either 7 or $49 \mathrm{~d}$ of age (10). Not surprisingly, therefore, the weekly administration of CYCLO did not normalize sterol metabolism in the lung and did not prevent the development of progressive "lipoid" pneumonitis. Relative organ weight, cholesterol content, and sterol synthesis (Fig. $1 D, J$, and $V$ ) in the lung all remain uncorrected. Despite treatment, there is progressive infiltration of the lung with lipid-laden macrophages (Fig. 5) and persistent, 
high relative mRNA levels for CD68 and CD11c (Fig. 2). As a result, by $160 \mathrm{~d}$ of age, these mutant mice develop a "lipoid" pneumonitis (Fig. $5 N$ ) that seems nearly identical to that seen in infants with NPC disease who die of progressive pulmonary failure (19).

In summary, these studies demonstrate that weekly CYCLO administration to the $n p c 1^{-/-}$mouse brings about the excretion of nearly all of the $\mathrm{C}$ that is sequestered in the various tissues during the preceding week and maintains the whole animal sterol pool at essentially normal levels. This therapeutic effect prevents the liver dysfunction seen in these animals and ameliorates the rate of neurodegeneration, but does not alter the progressive development of lung disease. Future studies must explore alternative means for delivering CYCLO directly into the CNS and lung. However, importantly, these studies also indicate that CYCLO administration is essentially free of side effects. The compound is rapidly cleared from the plasma into the urine without, apparently, causing any untoward effects. Even after $160 \mathrm{~d}$ of administration, there is no evidence of tissue damage so that liver and lung histology remain normal in the $n p c 1^{+/+}$animal. The only histological abnormality seen is mild vacuolization in some renal epithelial cells $49 \mathrm{~d}$ after beginning therapy, but even this lesion apparently does not progress.

Acknowledgments. We express our appreciation to Carolyn Crumpton, Mario Saucedo, Jennifer Burg, and S. Sean Campbell for their excellent technical assistance and to Annemarie Kelsey for expert preparation of the manuscript.

\section{REFERENCES}

1. Loftus SK, Morris JA, Carstea ED, Gu JZ, Cummings C, Brown A, Ellison J, Ohno K, Rosenfeld MA, Tagle DA, Pentchev PG, Pavan WJ 1997 Murine model of Niemann-Pick C disease: mutation in a cholesterol homeostasis gene. Science 277:232-235
2. Xie C, Turley SD, Pentchev PG, Dietschy JM 1999 Cholesterol balance and metabolism in mice with loss of function of Niemann-Pick $\mathrm{C}$ protein. Am J Physiol 276:E336-E344

3. Liu B, Xie C, Richardson JA, Turley SD, Dietschy JM 2007 Receptor-mediated and bulk-phase endocytosis cause macrophage and cholesterol accumulation in $\mathrm{Ni}$ emann-Pick C disease. J Lipid Res 48:1710-1723

4. Li H, Repa JJ, Valasek MA, Beltroy EP, Turley SD, German DC, Dietschy JM 2005 Molecular, anatomical, and biochemical events associated with neurodegeneration in mice with Niemann-Pick type C disease. J Neuropathol Exp Neurol 64:323-333

5. Gondré-Lewis MC, McGlynn R, Walkley SU 2003 Cholesterol accumulation in NPC1-deficient neurons is ganglioside dependent. Curr Biol 13:1324-1329

6. Beltroy EP, Liu B, Dietschy JM, Turley SD 2007 Lysosomal unesterified cholesterol content correlates with liver cell death in murine Niemann-Pick type $\mathrm{C}$ disease. J Lipid Res 48:869-881

7. Repa JJ, Li H, Frank-Cannon TC, Valasek MA, Turley SD, Tansey MG, Dietschy JM 2007 Liver X receptor activation enhances cholesterol loss from the brain, decreases neuroinflammation, and increases survival of the NPC1 mouse. J Neurosci 27:14470-14480

8. Posse De Chaves EI, Vance DE, Campenot RB, Kiss RS, Vance JE 2000 Uptake of lipoproteins for axonal growth of sympathetic neurons. J Biol Chem 275:1988319890

9. Liu B, Turley SD, Burns DK, Miller AM, Repa JJ, Dietschy JM 2009 Reversal of defective lysosomal transport in NPC disease ameliorates liver dysfunction and neurodegeneration in the npc1 $1^{-1-}$ mouse. Proc Natl Acad Sci USA 106:2377-2382

10. Liu B, Ramirez CM, Miller AM, Repa JJ, Turley SD, Dietschy JM 2010 Cyclodextrin overcomes the transport defect in nearly every organ of the newborn or mature NPC1 mouse leading to excretion of the sequestered cholesterol as bile acid. J Lipid Res 51:933-944

11. Davidson CD, Ali NF, Micsenyi MC, Stephney G, Renault S, Dobrenis K, Ory DS, Vanier MT, Walkley SU 2009 Chronic cyclodextrin treatment of murine NiemannPick C disease ameliorates neuronal cholesterol and glycosphingolipid storage and disease progression. PLoS One 4:e6951

12. Liu B, Li H, Repa JJ, Turley SD, Dietschy JM 2008 Genetic variations and treatments that affect the lifespan of the NPC1 mouse. J Lipid Res 49:663-669

13. Repa JJ, Turley SD, Quan G, Dietschy JM 2005 Delineation of molecular changes in intrahepatic cholesterol metabolism resulting from diminished cholesterol absorption. J Lipid Res 46:779-789

14. Valasek MA, Weng J, Shaul PW, Anderson RG, Repa JJ 2005 Caveolin-1 is not required for murine intestinal cholesterol transport. J Biol Chem 280:28103-28109

15. Xie C, Burns DK, Turley SD, Dietschy JM 2000 Cholesterol is sequestered in the brains of mice with Niemann-Pick type C disease but turnover is increased. J Neuropathol Exp Neurol 59:1106-1117

16. Zervas M, Dobrenis K, Walkley SU 2001 Neurons in Niemann-Pick disease type C accumulate gangliosides as well as unesterified cholesterol and undergo dendritic and axonal alterations. J Neuropathol Exp Neurol 60:49-64

17. Gould S, Scott RC 2005 2-Hydroxypropyl-beta-cyclodextrin (HP-beta-CD): a tox icology review. Food Chem Toxicol 43:1451-1459

18. Xie C, Turley SD, Dietschy JM 2000 Centripetal cholesterol flow from the extrahepatic organs through the liver is normal in mice with mutated Niemann-Pick type C protein (NPC1). J Lipid Res 41:1278-1289

19. Schofer O, Mischo B, Puschel W, Harzer K, Vanier MT 1998 Early-lethal pulmonary form of Niemann-Pick type $\mathrm{C}$ disease belonging to a second, rare genetic complementation group. Eur J Pediatr 157:45-49 